\title{
Patient Reported Frequency of Lupus Flare: Associations with Foundation Makeup and Sunscreen Use
}

\author{
Marline L. Squance ${ }^{1,2,3,4,5^{*}}$, Glenn E. M. Reeves ${ }^{1,3,4,5}$, John Attia1,4,5 \\ ${ }^{1}$ Faculty of Health and Medicine, University of Newcastle, Callaghan, Australia \\ ${ }^{2}$ Faculty of Science and Information Technology, University of Newcastle, Callaghan, Australia \\ ${ }^{3}$ Autoimmune Resource and Research Centre, New Lambton Heights, Australia \\ ${ }^{4}$ Hunter New England Health District, New Lambton Heights, Australia \\ ${ }^{5}$ Hunter Medical Research Institute, New Lambton Heights, Australia \\ Email: ${ }^{*}$ marline.squance@hnehealth.nsw.gov.au
}

Received 12 October 2014; revised 14 November 2014; accepted 3 December 2014

Copyright (C) 2014 by authors and Scientific Research Publishing Inc.

This work is licensed under the Creative Commons Attribution International License (CC BY). http://creativecommons.org/licenses/by/4.0/

(c) (i) Open Access

\begin{abstract}
Objective: To test the hypothesis that usage of foundation makeup (FM) and sunscreen lotion (SS), used individually or in combination, is associated with significant changes in the likelihood of lupus symptom exacerbation. Methods: Self-reported flare days (SRF) and use of FM and SS products, were retrospectively examined in 80 Caucasian Australian women with ACR classified SLE for a year. Negative binomial regression modelled SRF days (outcome) against independent FMSS variable and covariates: age; diagnosis years; outdoor hours; BMI; stress; immune therapy medication (ITM) use. Results: Statistically significant inverse associations between SRF days and FMSS use were found. Protective effects were statistically significant $(p<0.05)$ for combined FMSS exposure days (OR 0.998, CI 0.997 - 1.0) and FM alone (OR 0.603, CI 0.363 - 1.0). Significant associations consistent with increased SRF risk were seen in sub-analysis models for participants taking ITM: univariate model (OR 1.968, p = 0.03); multivariate model for FMSS (OR 2.11, CI 1.161 - 3.835); FM days (OR 1.855, CI 1.023 - 3.364). Results show SRF day reduction of $0.15 \%$ for each day of product exposure. Conclusion: Study results highlight protective effects of wearing FM with or without SS. This reduction in flare days ultimately has potential to improve quality of life in SLE patients.
\end{abstract}

\section{Keywords}

Lupus, Patient Perspective, Flare, Makeup, Sunscreen, Photosensitivity, Uv Protection, Immune Therapy Medications

\footnotetext{
${ }^{*}$ Corresponding author.
}

How to cite this paper: Squance, M.L., Reeves, G.E.M. and Attia, J. (2014) Patient Reported Frequency of Lupus Flare: Associations with Foundation Makeup and Sunscreen Use. Journal of Cosmetics, Dermatological Sciences and Applications, 4, 344-354. http://dx.doi.org/10.4236/jcdsa.2014.45046 


\section{Introduction}

Systemic Lupus Erythematosus (SLE) is a chronic autoimmune illness characterised by multi-system involvement that can be mild through to life threatening. SLE prevalence worldwide was recently estimated as 32 per 100,000 [1], with potential for underestimation due to milder cases not being diagnosed [2]-[4]. Community burden of SLE, particularly in industrialised western countries and Caucasian populations, is reported to be on the increase, which cannot be attributed to improved diagnostic criteria and investigations alone [4].

Environmental interactions along with intrinsic factors such as genetics, hormones and age have been reported as playing a role in SLE pathogenesis [5]-[7], with a small number of researchers suggesting that environmental exposures have a role in SLE progression and exacerbation patterns of sensitive individuals [5]-[10]. The interplay of endogenous and exogenous factors is thought to stimulate endocrine and immune regulatory systems interfering with immune tolerance [3] [9] [11]-[13], manifesting in either suppression or heightening of immune system responses.

The illness commonly exhibits periods of symptom quiescence and exacerbation (flare events). The unpredictable nature of SLE flares reduces quality of life and has the capacity to reduce functional ability [13]. The numbers of SLE specific risk assessment studies investigating relationships between illness exacerbation and everyday products are limited. Stimuli such as UV exposure, certain pharmaceutical compounds, hormones, infection and stress are the most researched and accepted triggers of flares [8] [14] [15]; however, in the majority of individuals, reasons for why and when flares occur remain unclear. Exposure to environmental agents including personal hygiene and grooming products, which regularly contain solvents, phthalates, parabens, perfumes, UV filters and pigments [16] [17], is suggested as potential flare triggers.

This study assesses the hypothesis that the use of common makeup products of foundation (FM) and sunscreen lotion (SS), used individually or in combination, is associated with significant changes in the likelihood of self-reported flares (SRF) of lupus symptoms.

\section{Patients and Methods}

This study was a retrospective analysis of a cohort of 80 Caucasian female SLE patients as defined by the American College of Rheumatology (ACR) classification criteria for SLE [18]. Participants completed study specific questionnaires and interview to examine their flare experience and exposure to environmental products within daily living practices as part of a wider pilot study, "The Environmental Determinants of Lupus Flare (EDOLF)". Data were of a self-reported nature over a 12 month study period capturing a full seasonal cycle.

Methods have been reported previously in detail [19] and are briefly summarized here with methods specific to exposure and usage of foundation makeup (FM) and sunscreen (SS) outlined. Ethical review and approval according to Declaration of Helsinki 2008 [20] was granted from the University of Newcastle and Hunter New England Health Human Research Ethics Committees.

\subsection{Study Population}

Participants were recruited through the Autoimmune Resource and Research Centre (ARRC) and private Immunology clinics in the Hunter/Central Coast region of New South Wales, Australia. SLE diagnosis was confirmed through a health record audit to establish SLE diagnosis adhered to the ACR SLE Classification guidelines [18]. Due to minimal numbers of males meeting the ACR criteria, the final study cohort was limited to females only. The population was of a homogeneous Caucasian background.

\subsection{Data Collection}

Participants completed study specific questionnaires for assessment of medical history, home environment, and lifestyle practices as part of the wider EDOLF study. Of particular interest were participant patterns of commercial product usage associated with personal hygiene and household cleaning practises. Participants also attended the ARRC for a clinical appointment where standard measures of health and their SLE flare history experience (SRF) were documented.

\subsubsection{Product Exposure Assessment}

Participants completed a Home Cleaning and Maintenance Product list (HCMPL) documenting all products used 
within their home for cleaning and for personal care. The HCMPL collected the following product information: product type description, intended purpose of use, brand names and product usage over the study year. Exposure was calculated by combining count values of self-reported use of each product within a scale of "daily", "weekly", "monthly", "yearly" and "don't know".

The study goal was to examine common product use patterns and flare events; therefore, the developed methods did not direct participants to particular products or product categories. The HCMPL instructed participants to document all products used and stored within cupboards in various housing rooms (bathroom, kitchen, laundry, garages, etc.). Participants self-selected the products they documented without independent visual confirmation that all products had been documented.

Product groups were categorised based on intended purpose of named product. The product category of "makeup" (FMSS) consisted of topical lotions of FM and SS only. Other makeup products such as lipsticks, eye shadow, mascara and colouring agents such as blush were self-reported by a small number of participants only, so they were not included within this analysis. As a crosscheck technique for products of specific interest, participants were asked questions within the primary study questionnaire regarding FM and SS and their regime of use.

Days of FMSS exposure was calculated for each participant as a total exposure day count by collating selfreported product/chemical exposure activity information and the specific product question responses from the primary study questionnaire. Duplication of individual products and usage day counts was avoided by crosschecking data from each source.

\subsubsection{Patient Reported Flare Outcomes}

Flare assessment processes were not administered by a physician within a clinical review appointment, therefore traditional tools for assessing disease activity (e.g., SLEDAI) could not be used. To capture the lived experience of lupus, study flare event history was taken directly from the individual participant perspective and used standardisation scripted structured flare history interview [19] inclusive of a novel flare definition. The flare event definition chosen reflected a chronic autoimmune illness characterised by relapsing and remitting flare patterns whilst also incorporating the concept of symptom stability, punctuated by sustained exacerbation.

An exacerbation is defined as: The appearance of a new clinical sign/symptom or the clinical worsening of a previous sign/symptom that had been stable for at least the previous 30 days and which persisted for a minimum of 24 hours [21].

SRF days were a calculation of self-reported number and average length of SRF events reported in the study period. Final analysis did not include participants that reported a "constant" state of flaring.

\subsubsection{Risk Factors}

Study specific questionnaires completed included information on participant demographic, medical history, general health and wellbeing. Each participant nominated an approximate date of their SLE diagnosis which was crosschecked within the health record audit phase of the study. Participant self-assessment of their socio economic status (SES) of "Above Australian Average", "Australian Average" and "Below Australian Average" were recorded. Stress levels were recorded via a visual analogue scale (VAS) of 0-100 with end points of "Not stressed at all" and "Highly stressed". Participant body mass index (BMI) was calculated and coded according to Australian Government Health Guidelines categories of "underweight (<18.5)", "normal weight (18.5 - 24.99)", "overweight (25 - 29.99)" and "Obese (>30)". Study period hours spent outdoors were calculated from participants' nominated hours for each weekday and weekend day. Current smoking status was captured as a dichotomised "yes", "no" response.

Immune modulating and suppressant medications (immune therapy medications, ITM): Methotrexate, Hydroxychloroquine, Prednisolone, Azathioprine, intravenous immunoglobulin, Dapsone and Mycophenolate were categorised as a single group to examine effect on SRF history in relation to FMSS use. Vitamin D supplementation, whilst considered by many to have properties of immune modulation [22]-[25], was considered separately.

\subsection{Statistical Methods}

Due to over dispersion, a negative binomial regression model was used to assess the relationship between SRF days and FMSS usage days. Covariates considered were: participant age; diagnosis years; outdoors hours; SES; 
BMI; stress; Vitamin D, hormones and ITM use.

Initially, all variables of interest were included in the model with a backward stepwise approach to identify the best possible multivariate model. Interactions were tested for SRF days and covariates with significant effects at the $<0.05$ level retained in the model. All normality assumptions were verified by inspection of probability plots and histograms of residuals. Associations were expressed as odds ratios with $95 \%$ confidence intervals. All analyses were performed with the use of STATA v11.0 (StataCorp LP, College Station, Texas, USA).

\section{Results}

A total of 159 personal health records were audited to confirm a SLE diagnosis based upon documented evidence of the 11 ACR classification criteria [18]. Of these, 83 participants met the classification criterion of $\geq 4$ out of 11, and completed all components of the study. The structured interview process resulted in 3 participants reporting a SRF history of "constant flaring"; therefore these participants were excluded from final analysis. Health record audit showed a broad representation of SLE clinical manifestations with clinical features of malar rash, photosensitivity, arthritis, and presence of antinuclear antibodies being frequently documented.

Table 1 represents the distribution of characteristics across the final 80 SLE participants. Participants' mean age was 47.69 years and diagnosed with SLE for approximately 2 - 38 years (mean 7.7 years). The participant

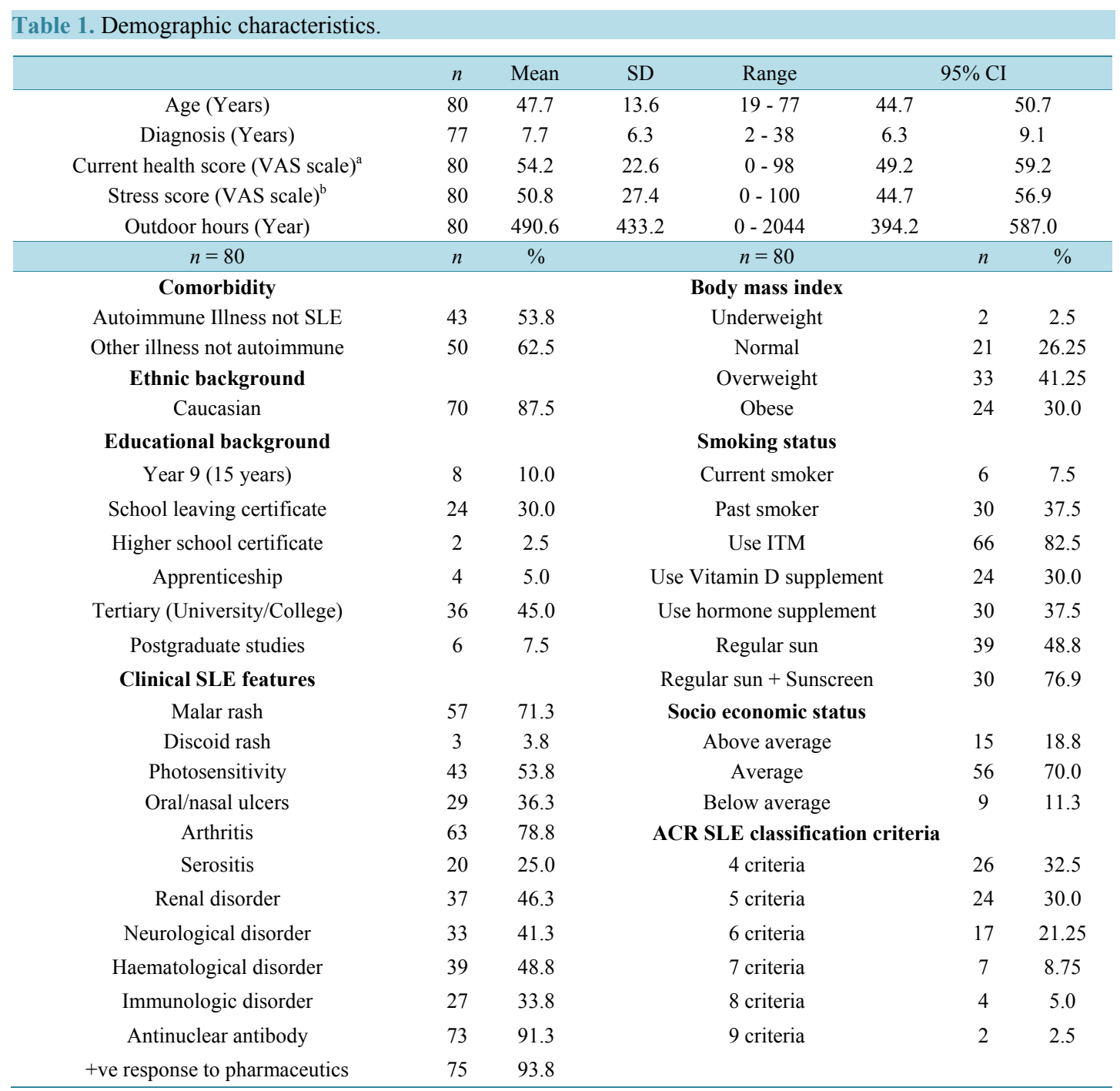

${ }^{\mathrm{a}}$ Visual Analogue Scale Score of 0 - 100—Current Health (0) Extremely Poor—(100) Excellent; ${ }^{\mathrm{b}}$ Stress (0) Not stressed at all-(100) Highly stressed. 
inclusion criteria did exclude persons with a diagnosis history less than 2 years duration. Over $62.5 \%$ of participants self-reported comorbidities. The majority of participants were not current smokers ( $92.5 \%)$ however, a larger number reported being past smokers (37.5\%). Current smoking status was confirmed by serum cotinine assay and showed concordant results with self-report.

The analysis also included data on the usage of ITM as well as supplementation of Vitamin D and hormonal treatments. Participant use of ITM was common (82.5\%) with many being prescribed 2 or more ITMs. No assessment of individual participant medication compliance was undertaken.

Table 2 represents data in specific reference to SRF events and makeup product usage. Tabulated results showed that participants experienced a mean of $6.8 \mathrm{SRF}$ events within the study year ranging from 0 flare events to 1 event per week. Counts of SRF days ranged 0 - 240 days with a mean count of 29.2 SRF days. Three participants reported single events that equated to 60 - 90 SRF day counts. FM was used by $73.75 \%$ of the cohort and SS was used by $77.5 \%$. Many participants used more than one FMSS product each day. SS usage in regards to regular sun exposure was explored with $49 \%$ reporting regular sun exposure (Table 1) and of those $76.9 \%$ responded as always wearing SS when in the sun. A range of 0 - 1223 (mean 291) total exposure days for overall FMSS category indicates multiple individual product usage within the study year.

Negative binomial regression was used to model SRF days (outcome) against independent FMSS product variables as well as social, lifestyles and illness variables with results summarised within Table 3. Statistically

Table 2. Self-reported flare (SRF) and makeup product (FMSS) usage.

\begin{tabular}{ccccccc}
\hline & $n$ & Mean & SD & Range & \multicolumn{2}{c}{$95 \%$ CI } \\
\hline SRF no. (Year) & 80 & 6.8 & 9.7 & $0-52$ & 4.6 & 8.9 \\
SRF days (Year) & 80 & 29.2 & 39 & $0-240$ & 20.5 & 37.9 \\
FMSS exposure days & 80 & 291.1 & 214.2 & $0-1223$ & 243.4 & 338.7 \\
FMSS product count & 80 & 1.6 & 0.9 & $0-7$ & 1.4 & 1.8 \\
$n=80$ & $n$ & $\%$ & & & & \\
Use of FM & 59 & 73.8 & & & & \\
Use of SS & 62 & 77.5 & & & & \\
\hline
\end{tabular}

Table 3. Negative binomial regression for flare days $(\mathrm{Fc})$ and independent variables.

\begin{tabular}{ccccc}
\hline & OR & $\mathrm{p}>|\mathrm{Z}|$ & \multicolumn{3}{c}{$95 \% \mathrm{CI}$} \\
\hline Univariate model (Fc) & & & & \\
\hline FMSS product days & 0.998 & $\mathbf{0 . 0 1}$ & 0.998 & 3.0 \\
\hline Immune therapy medications & 1.968 & $\mathbf{0 . 0 3}$ & 1.088 & 0.56 \\
FM & 0.58 & $\mathbf{0 . 0 4}$ & 0.35 & 1.40 \\
SS & 0.79 & 0.43 & 0.45 & 2.45 \\
Vitamin D & 1.51 & 0.10 & 0.93 & 1.94 \\
Hormone treatment & 1.22 & 0.39 & 0.78 & 1.00 \\
Age & 0.99 & 0.06 & 0.97 & 1.00 \\
Years of diagnosis & 0.97 & 0.07 & 0.93 & 5.41 \\
Body mass index & 1.23 & 0.78 & 0.28 & 3.73 \\
Smoking & 1.61 & 0.27 & 0.69 & 1.01 \\
Stress & 1.00 & 0.63 & 0.99 & 1.00 \\
Outdoor hours & 1.0 & 0.10 & 1.0 & 1.45 \\
Regular sun & 0.93 & 0.74 & 0.59 & 1.0 \\
\hline Multivariate model (Fc) & & & & 3.835 \\
FMSS product days & 0.998 & $\mathbf{0 . 0 0}$ & 0.997 & 1.0 \\
Immune therapy medication & 2.11 & $\mathbf{0 . 0 1}$ & 1.161 & 0.363 \\
FM days & 0.603 & $\mathbf{0 . 0 5}$ & 0.023 & $\mathbf{0 . 0 4}$ \\
\hline Immune therapy medication & 1.855 & & 1.03 & \\
\hline
\end{tabular}


significant associations for independent variables were found for FMSS product days, ITM and FM use; but not for SS as a sub group analysis of the FMSS product days. The analysis was adjusted for age, diagnosis years, SES, BMI, smoking, stress, therapeutic supplements of vitamin D and hormone treatments, sunburn events, and outdoor hours in the study year. No confounding effect was found for any of these factors.

Protective effects, as demonstrated by odds ratio values, were statistically significant on both univariate and multivariate analysis of FMSS product exposure days (OR 0.998, CI 0.997 - 1.0) and FM usage (OR 0.603, CI 0.363 - 1.0). Surprisingly, significant associations consistent with increased SRF risk were seen in a univariate model for participants undertaking ITM $(\mathrm{OR} 1.968, \mathrm{p}=0.03)$. This ITM association was also retained within multivariate models for FMSS product days (OR 2.11, CI 1.161 - 3.835) and the sub analysis of FM days (OR 1.855 , CI $1.023-3.364)$. This is likely a reflection of selection bias in that those with more severe SLE are more likely to be prescribed ITM.

Participant total SRF days as a function of FMSS exposure (with 95\% CI) within the study year for participants undertaking ITM and also for those that are not on ITM are presented in Figure 1. A reduction of SRF days with usage of FMSS products was demonstrated, and was consistent regardless of the participant taking ITM or not. There were significantly more SRF days in individuals on ITM $(p=0.01)$ however overall reduction of SRF days with increased chemical exposure was not contingent upon ITM therapy. The model estimates that an SLE patient on ITM can potentially have reduction in SRF days experienced within a year by a factor of $0.0015(0.15 \%)$ with each day of increasing FMSS product exposure. Absolute FMSS product exposure ranges from 0 to $\sim 600$ exposure days: for every 100 days of additional FMSS product exposure, the lupus SRF incidence rate drops by $14 \%$. Across the exposure range, the risk drops by 59\%-putting this in terms of risk reduction, the SRF incidence rate for FMSS exposure level equal to 600 days is over half that observed with an exposure level equal to 0 .

\section{Discussion}

This study found a statistically significant inverse association between SRF days and exposure to FMSS products defined as FM and SS. Results show those SRF days were reduced by a factor of $0.15 \%$ for each day of FMSS product exposure over the study year, indicating a protective component to FMSS product usage. The sub analysis of FMSS category with ITM usage indicates that the reduction of SRF days was not contingent on ITM use. This reduction in symptom exacerbation days ultimately has potential to improve quality of life in SLE patients.

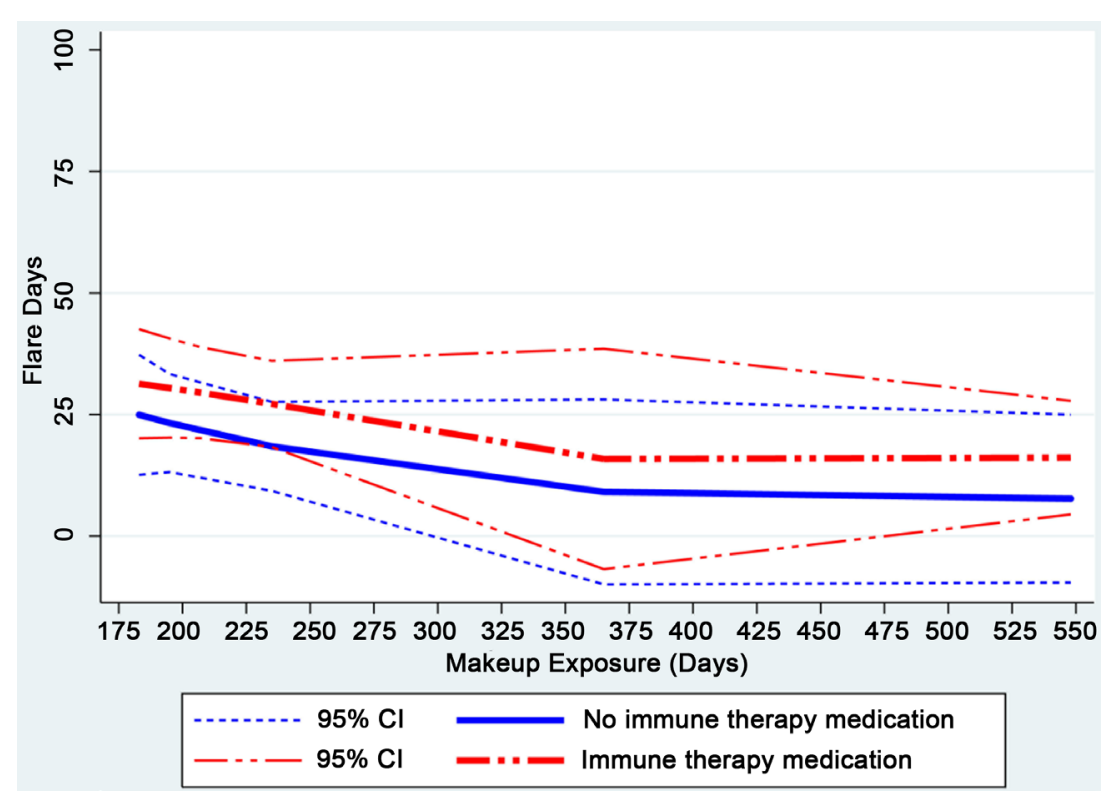

Figure 1. Self-reported flare (SRF) day events as a function of FMSS exposure days and immune therapy medication use for the study year, together with $95 \%$ confidence bands. 
SLE flare days within this study were calculated from the lived health experience of the individual participant rather than clinical markers of symptom exacerbation. Total flare days were calculated using the number and average length of SRF in days. No assessments of SRF severity in regards to health and quality of life impacts were made; however, 3 participants recalled long periods of symptom activation and recovery of 60 - 90 days. Due to the retrospective nature of reporting, symptom exacerbation characteristics specific to a reported single SRF were not explored. However, the reported tendency of lupus patients to inaccurately attribute symptom fluctuations to flare events [26]-[29] would tend to create bias against detection of a significant association with product exposure.

Within the study group, symptoms of malar and body rash, photosensitivity, joint pain and fatigue were widely reported as common flare symptoms (Table 1) and they also had a high impact on quality of life and body self-image. The health record audit found that the commonest symptoms included joint involvement (arthritis $78.75 \%)$, malar rash $(71.25 \%)$ and photosensitive reactions (54\%). These rates are similar to other studies examining epidemiology of SLE and symptom exacerbation [2] [6] [14] [30] [31]; however, the reported rates of malar rash within these studies are lower than that found within our Australian study population. This could be a result of the study involving a homogeneous Caucasian population; UV exposure intensity within the Australian environment; and the high prescribed usage of a known photosensitizing pharmaceutical, Hydroxychloroquine, as a primary treatment within the study population.

ITM usage (82.5\%) was considered a potential confounder of the impact of FMSS product use; unexpectedly, our results indicate that ITM use presented an elevated risk of SRF activity (OR 2.11). It is likely that this is a reflection of selection bias in that those with more severe SLE illness experience more flare days as a result of flare frequency and intensity, requiring a management regime involving one or more ITM.

SLE skin symptoms of malar rash, scarring and photosensitive reactions result as either an illness manifestation or pharmaceutical therapy side effect [32]. To better manage these symptoms and their impacts, clinicians and health educators offer self-management advice to adopt lifestyle practices that focus on sun protection measures [30] [33]-[35] and, if necessary, masking of skin irregularities through the application of physical disguises such as makeup [36] [37]. Advice on reduction of UV exposure includes avoidance of long periods of UV exposure, wearing hats and sun protective clothing and regular topical application of physical and chemical UV filters and barriers in the form of lotions.

Adherence to the use of sun protection in the form of SS use has been widely examined and it has been argued to be a sometimes ineffective sun protective measure due to the practise of selective use only when significant exposure activity is planned. This sporadic use can in fact lead to higher UV exposure [38] [39] from prolonged time periods spent within direct sunlight and other UV sources in living and work environments. Repeated mini exposures to UV radiation occurring from sunlight sources and from internal lighting systems of modern homes, offices and places of daily activity, has been estimated at as much as $80 \%$ of total lifetime exposure [32] leaving a photosensitive person vulnerable to adverse effects. The amount of incidental lifetime UV exposure indicates that daily application of visually appealing products containing UV protection capabilities as a prophylactic measure of UV protection may be more effective particularly in a photosensitive population.

Vila et al. [35], in a SLE and sunlight exposure study, found many clinical outcomes significantly improved with use of photo protection measures. Specifically, patients who adhered to UV protective regimes had significantly lower renal involvement, thrombocytopenia, hospitalisations, and reduced requirement for either increased dosage or additional ITM. Our study, whilst not finding a significant association with the use of SS alone and SRF, did find health improvements in the form of reduced SRF days, with the use of FM alone as well as FMSS as a combined product group.

SS use within the study group was reported at $77.5 \%$ which is double that of reported rates of use within the Australian NSW Health adult female population (34.6\%) [40]. Comparison rates for the use of FM and SS across other SLE studies was limited due to the small number of studies; however, there are reported rates of $50 \%$ SS use within SLE patients in Puerto Rico [35], and 54.7\% SS and 60.4\% cosmetic use in a US based general population birth mothers study [41]. These rates suggest that our study population was more compliant with sun protection measures than the general Australian population or other international study populations.

Commercial personal product manufacturers have increased the use of UV protective chemicals and physical barriers to improve product saleability and acceptability [30] [42]. UV protectors in products are commonly in the form of organic and physical inorganic chemicals such as Titanium Dioxide $\left(\mathrm{TiO}_{2}\right)$, Zinc oxides $(\mathrm{ZnO})$ and mineral pigments including mica and other oxides, which act to either block, reflect or scatter UVA and UVB 
radiation limiting penetration. A wide range of personal care products label these agents within their product ingredients. Everyday products of shampoos, shower gels, face moisturisers, soaps, clothing, cleaning agents as well as common makeup products such as foundation makeup, contain at least one UV protective agent [31] [43].

The NHANES study reports that $96.8 \%$ of randomly selected urine samples were found to contain a UV protective chemical, Benzophenone-3, indicating widespread exposure to UV protectors as a result of daily activities. It is possible that the protective and significant association with FM and SS found in our study could be associated with the individual participant's chemical use of other commercial agents, including undisclosed personal cosmetic and cleansing products. The ubiquitous use of UV filters and UV protection chemicals in everyday cleaning and personal products could also result in additional unintentional UV protection via daily chores and hygiene habits [44].

It is also proposed that the reported positive self-image that occurs with cosmetic coverage of disfiguring symptoms, such as malar rash, scarring and photosensitivity [30] [36] [37] [45] could also have resulted in improved psychological wellbeing and quality of life within those participants and therefore reduced impacts of SLE symptoms and recorded flare days.

\section{Study Strengths and Weaknesses}

This study demonstrated that SRF day was reduced in this SLE cohort with exposure to FMSS products of FM and SS. This apparent protective aspect of FMSS product use was detected regardless of whether they were taking ITM as part of their illness management regime. The study provides pilot data within the context of a retrospective study that relies on self-reported participant assessment of exposure and flare events in a Caucasian population. The capacity to assess individual measures of actual exposure or day counts of symptom exacerbation as defined as a "flare" event was not possible at this time. The lack of ethnic diversity within our Australian study population would reduce the capacity for extrapolation of results to the wider, more diverse international SLE population.

Flare assessment was undertaken from a patient perspective with adherence to a strict protocol for documentation; however, confirmation from clinical data did not occur. Hence, flare days were estimated across the study year based upon participant self-report and this may have resulted in an overestimation of actual days. However, this overestimation would be expected to create a bias against detection of a significant association with product exposure.

Measurement bias within the assessment of product exposure process was minimised by adopting a process of crosschecking reported usage within two separate data collection tools (HCMPL and study specific questionnaire). The FMSS product category did not include other cosmetic and personal products that may contain sunscreen agents, so it is possible that the effect we are noting within our study could be attributed in part to other products used routinely. It is unlikely that the reported findings represent recall bias as patients were asked about FM and SS use in the context of a long list of products with no indication of associations being tested.

\section{Conclusions}

This study has examined the effects of FMSS exposure and exacerbation of SLE symptoms as defined by "SRF days". The study results have highlighted a protective effect of wearing FMSS products, in particular FM with or without additional SS products. However, this study has not demonstrated a conclusive link to any particular product chemical component due to the inability to refine: individual product chemical content or concentrations, or the potential effects of product chemical admixing.

The study results indicate that the investigation of SLE illness behaviour in respect to everyday beauty products needs further exploration. Formulations and concentrations of product chemical components change readily to meet consumer and commercial demands reducing capacity for researchers to gauge exact causation links between chemical exposure and health risk.

\section{Acknowledgements}

This study forms part of the Environmental Determinants of Lupus Flare (EDOLF) PhD study, University of Newcastle. The authors would like to thank Associate Professor Howard Bridgman for his guidance in the writ- 
ing of this paper, the study participants and also Dr. Patrick McElduff, Dr. Maya Guest and Dr. May Boggess for their help with statistical analysis and graphing methods.

The study was supported by the Autoimmune Resource Research Centre (Not-for-profit charity www.autoimmune.org.au), and the Val Badham Research Scholarship for Immunology, University of Newcastle Foundation. The Authors declare that there is no conflict of interest.

\section{References}

[1] Hayter, S.M. and Cook, M.C. (2012) Updated Assessment of the Prevalence, Spectrum and Case Definition of Autoimmune Disease. Autoimmunity Reviews, 11, 754-765. http://dx.doi.org/10.1016/j.autrev.2012.02.001

[2] Danchenko, N., Satia, J.A. and Anthony, M.S. (2006) Epidemiology of Systemic Lupus Erythematosus: A Comparison of Worldwide Disease Burden. Lupus, 15, 308-318. http://dx.doi.org/10.1191/0961203306lu2305xx

[3] Cooper, G.S. and Stroehla, B.C. (2003) The Epidemiology of Autoimmune Diseases. Autoimmunity Reviews, 2, 119125. http://dx.doi.org/10.1016/S1568-9972(03)00006-5

[4] Jacobson, D.L., Gange, S.J., Rose, N.R. and Graham, N.M. (1997) Epidemiology and Estimated Population Burden of Selected Autoimmune Diseases in the United States. Clinical Immunology and Immunopathology, 84, 223-243. http://dx.doi.org/10.1006/clin.1997.4412

[5] Pons-Estel, G.J., Alarcon, G.S., Scofield, L., Reinlib, L. and GS, C. (2010) Understanding the Epidemiology and Progression of Systemic Lupus Erythematosus. Seminars in Arthritis and Rheumatism, 39, 257-268. http://dx.doi.org/10.1016/j.semarthrit.2008.10.007

[6] Dooley, M.A. and Hogan, S.L. (2003) Environmental Epidemiology and Risk Factors for Autoimmune Disease. Current Opinion in Rheumatology, 15, 99-103. http://dx.doi.org/10.1097/00002281-200303000-00002

[7] Foster, M.W. and Aston, C.E. (2003) A Practice Approach for Identifying Previously Unsuspected Environmental Contributors to Systemic Lupus Erythematosus and Other Complex Diseases. Environmental Health Perspectives, 111, 593-597. http://dx.doi.org/10.1289/ehp.5665

[8] Zandman-Goddard, G., Solomon, M., Rosman, Z., Peeva, E. and Shoenfeld, Y. (2012) Environment and Lupus-Related Diseases. Lupus, 21, 241-250. http://dx.doi.org/10.1177/0961203311426568

[9] Mayes, M.D. (1999) Epidemiologic Studies of Environmental Agents and Systemic Autoimmune Diseases. Environmental Health Perspectives, 107, 743-748. http://dx.doi.org/10.1289/ehp.99107s5743

[10] Hess, E.V. (1999) Are There Environmental Forms of Systemic Autoimmune Diseases? Environmental Health Perspectives, 107, 709-711. http://dx.doi.org/10.1289/ehp.99107s5709

[11] Cooper, G.S., Gilbert, K.M., Greidinger, E.L., James, J.A., Pfau, J.C., Reinlib, L., et al. (2008) Recent Advances and Opportunities in Research on Lupus: Environmental Influences and Mechanisms of Disease. Environmental Health Perspectives, 116, 695-702. http://dx.doi.org/10.1289/ehp.11092

[12] Gourley, M. and Miller, F.W. (2007) Mechanisms of Disease: Environmental Factors in the Pathogenesis of Rheumatic Disease. Nature Reviews Rheumatology, 3, 172-180. http://dx.doi.org/10.1038/ncprheum0435

[13] Cooper, G.S., Treadwell, E.L., St. Clair, E.W., Gilkeson, G.S. and Dooley, M.A. (2007) Sociodemographic Associations with Early Disease Damage in Patients with Systemic Lupus Erythematosus. Arthritis Care and Research, 57, 993-999. http://dx.doi.org/10.1002/art.22894

[14] Cooper, G.S., Wither, J., Bernatsky, S., Claudio, J.O., Clarke, A., Rioux, J.D., et al. (2010) Occupational and Environmental Exposures and Risk of Systemic Lupus Erythematosus: Silica, Sunlight, Solvents. Rheumatology (Oxford), 49, 2172-2180. http://dx.doi.org/10.1093/rheumatology/keq214

[15] Hess, E.V. (2002) Environmental Chemicals and Autoimmune Disease: Cause and Effect. Toxicology, 181-182, 65-70.

[16] Dodson, R.E., Nishioka, M., Standley, L.J., Perovich, L.J., Brody, J.G. and Rudel, R.A. (2012) Endocrine Disruptors and Asthma-Associated Chemicals in Consumer Products. Environmental Health Perspectives, 120, 935-943. http://dx.doi.org/10.1289/ehp.1104052

[17] Altman, R.G., Morello-Frosch, R., Brody, J.G., Rudel, R., Brown, P. and Averick, M. (2008) Pollution Comes Home and Gets Personal: Women's Experience of Household Chemical Exposure. Journal of Health and Social Behavior, 49, 417-435. http://dx.doi.org/10.1177/002214650804900404

[18] Rheumatology ACo (2005) 1997 Update of the 1982 American College of Rheumatology Revised Criteria for Classification of Systemic Lupus Erythematosus. Classification Criteria for Diagnosis of Systemic Lupus Erythematosus.

[19] Squance, M.L., Guest, M., Reeves, G., Attia, J. and Bridgman, H. (2014) Exploring Lifetime Occupational Exposure and SLE Flare: A Patient-Focussed Pilot Study. Lupus Science \& Medicine, 1, e000023.

[20] Association, W.M. (2008) World Medical Association Declaration of Helsinki. 59th WMA General Assembly, Seoul, 
October 2008. www.wma.net/en/30publications/10policies/b3/17c.pdf

[21] Poser, C.M., Paty, D.W., Scheinberg, L., McDonald, W.I., Davis, F.A., Ebers, G.C., et al. (1983) New Diagnostic Criteria for Multiple Sclerosis: Guidelines for Research Protocols. Annals of Neurology, 13, 227-231. http://dx.doi.org/10.1002/ana.410130302

[22] Borges, M.C., Martini, L.A. and Rogero, M.M. (2011) Current Perspectives on Vitamin D, Immune System, and Chronic Diseases. Nutrition, 27, 399-404. http://dx.doi.org/10.1016/j.nut.2010.07.022

[23] Guillot, X., Semerano, L., Saidenberg-Kermanac'h, N., Falgarone, G. and Boissier, M.C. (2010) Vitamin D and Inflammation. Joint Bone Spine, 77, 552-557. http://dx.doi.org/10.1016/j.jbspin.2010.09.018

[24] Parravicini, V. and Caserta, S. (2010) The Immunomodulatory Roles of Vitamin D: New Tricks for an Old Dog. Molecular Interventions, 10, 204-208. http://dx.doi.org/10.1124/mi.10.4.3

[25] Zhang, H.L. and Wu, J. (2010) Role of Vitamin D in Immune Responses and Autoimmune Diseases, with Emphasis on its Role in Multiple Sclerosis. Neuroscience Bulletin, 26, 445-454. http://dx.doi.org/10.1007/s12264-010-0731-8

[26] Carr, F.N., Nicassio, P.M., Ishimori, M.L., Moldovan, I., Katsaros, E., Torralba, K., et al. (2011) Depression Predicts Self-Reported Disease Activity in Systemic Lupus Erythematosus. Lupus, 20, 80-84.

http://dx.doi.org/10.1177/0961203310378672

[27] Griffiths, B., Mosca, M. and Gordon, C. (2005) Assessment of Patients with Systemic Lupus Erythematosus and the Use of Lupus Disease Activity Indices. Best Practice \& Research Clinical Rheumatology, 19, 685-708. http://dx.doi.org/10.1016/j.berh.2005.03.010

[28] Leong, K.P., Chong, E.Y., Kong, K.O., Chan, S.P., Thong, B.Y., Lian, T.Y., et al. (2010) Discordant Assessment of Lupus Activity between Patients and Their Physicians: The Singapore Experience. Lupus, 9, 100-106. http://dx.doi.org/10.1177/0961203309345748

[29] Yen, J.C., Abrahamowicz, M., Dobkin, P.L., Clarke, A.E., Battista, R.N. and Fortin, P.R. (2003) Determinants of Discordance between Patients and Physicians in Their Assessment of Lupus Disease Activity. The Journal of rheumatology, 30, 1967-1976.

[30] Obermoser, G. and Zelger, B. (2008) Triple Need for Photoprotection in Lupus Erythematosus. Lupus, 17, 525-527. http://dx.doi.org/10.1177/0961203308089440

[31] Wang, J., Kay, A.B., Fletcher, J., Formica, M.K. and McAlindon, T.E. (2008) Is Lipstick Associated with the Development of Systemic Lupus Erythematosus (SLE)? Clinical Rheumatology, 27, 1183-1187. http://dx.doi.org/10.1007/s10067-008-0937-6

[32] Ting, W.W. and Sontheimer, R.D. (2001) Local Therapy for Cutaneous and Systemic Lupus Erythematosus: Practical and Theoretical Considerations. Lupus, 10, 171-184. http://dx.doi.org/10.1191/096120301667674688

[33] Ilchyshyn, L., Hawk, J.L. and Millard, T.P. (2008) Photoprotection: Does It Work? Lupus, 17, 705-707. http://dx.doi.org/10.1177/0961203308093924

[34] Kuhn, A., Gensch, K., Haust, M., Meuth, A.M., Boyer, F., Dupuy, P., et al. (2011) Photoprotective Effects of A Broad-Spectrum Sunscreen in Ultraviolet-Induced Cutaneous Lupus Erythematosus: A Randomized, Vehicle-Controlled, Double-Blind Study. Journal of the American Academy of Dermatology, 64, 37-48. http://dx.doi.org/10.1016/j.jaad.2009.12.053

[35] Vila, L.M., Mayor, A.M., Valentin, A.H., Rodriguez, S.I., Reyes, M.L., Acosta, E., et al. (1999) Association of Sunlight Exposure and Photoprotection Measures with Clinical Outcome in Systemic Lupus Erythematosus. Puerto Rico Health Sciences Journal, 18, 89-94.

[36] Boehncke, W.H., Ochsendorf, F., Paeslack, I., Kaufmann, R. and Zollner, T.M. (2002) Decorative Cosmetics Improve the Quality of Life in Patients with Disfiguring Skin Diseases. European Journal of Dermatology, 12, 577-580.

[37] Shear, N.H. and Graff, L. (1987) Camouflage Cosmetics in Dermatologic Therapy. Canadian Family Physician, 33, 2343-2346.

[38] Autier, P., Boniol, M. and Dore, J.F. (2007) Sunscreen Use and Increased Duration of Intentional Sun Exposure: Still a Burning Issue. International Journal of Cancer, 121, 1-5. http://dx.doi.org/10.1002/ijc.22745

[39] Manova, E., von Goetz, N., Hauri, U., Bogdal, C. and Hungerbuhler, K. (2012) Organic UV Filters in Personal Care Products in Switzerland: A Survey of Occurrence and Concentrations. International Journal of Hygiene and Environmental Health, 216, 508-514.

[40] NSW Government (2011) Research CfEa. New South Wales Population Health Survey 2010. Centre for Epidemiology and Research PHD, NSW Department of Health, Australia.

[41] Schlumpf, M., Kypke, K., Wittassek, M., Angerer, J., Mascher, H., Mascher, D., et al. (2010) Exposure Patterns of UV Filters, Fragrances, Parabens, Phthalates, Organochlor Pesticides, PBDEs, and PCBs in Human Milk: Correlation of UV Filters with Use of Cosmetics. Chemosphere, 81, 1171-1183. http://dx.doi.org/10.1016/j.chemosphere.2010.09.079 
[42] Nohynek, G.J., Lademann, J., Ribaud, C. and Roberts, M.S. (2007) Grey Goo on the Skin? Nanotechnology, Cosmetic and Sunscreen Safety. Critical Reviews in Toxicology, 37, 251-277. http://dx.doi.org/10.1080/10408440601177780

[43] NLM (2013) Household Products Database. http://householdproducts.nlm.nih.gov/index.htm

[44] Calafat, A.M., Wong, L.Y., Ye, X., Reidy, J.A. and Needham, L.L. (2008) Concentrations of the Sunscreen Agent Benzophenone-3 in Residents of the United States: National Health and Nutrition Examination Survey 2003-2004. Environmental Health Perspectives, 116, 893-897. http://dx.doi.org/10.1289/ehp.11269

[45] Hale, E.D., Treharne, G.J., Norton, Y., Lyons, A.C., Douglas, K.M., Erb, N., et al. (2006) “Concealing the Evidence”: The Importance of Appearance Concerns for Patients with Systemic Lupus Erythematosus. Lupus, 15, 532-540. http://dx.doi.org/10.1191/0961203306lu2310xx 
Scientific Research Publishing (SCIRP) is one of the largest Open Access journal publishers. It is currently publishing more than 200 open access, online, peer-reviewed journals covering a wide range of academic disciplines. SCIRP serves the worldwide academic communities and contributes to the progress and application of science with its publication.

Other selected journals from SCIRP are listed as below. Submit your manuscript to us via either submit@scirp.org or Online Submission Portal.
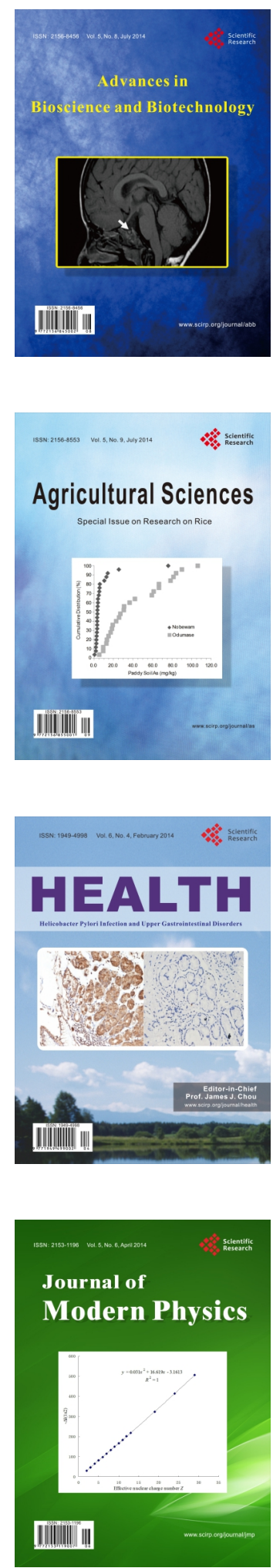
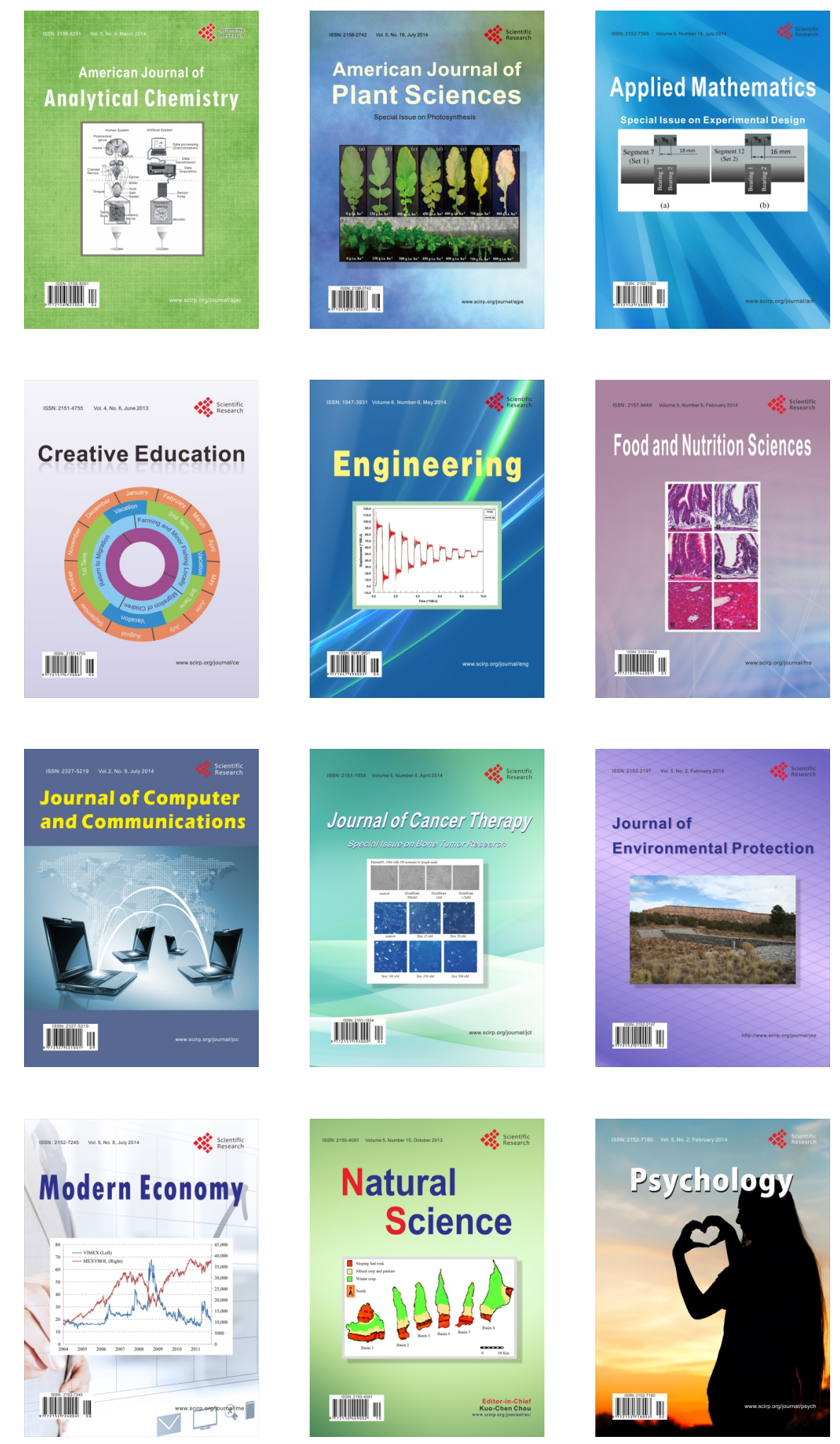